\title{
Performance Analysis of Opportunistic Routing in Multi-Sink Mobile Ad Hoc Wireless Sensor Networks
}

\author{
Amandeep Singh Dhaliwal \\ Department of Computer Science, \\ Punjabi University, Patiala
}

\author{
Maninder Singh \\ Department of Computer Science \\ Punjabi University, Patiala
}

\begin{abstract}
Wireless Sensor Networks are an active research area in diverse fields like military surveillance, habitat monitoring, weather and others. In WSN, the sensor nodes have a limited transmission range, processing, storage capabilities and energy resources. In this paper we illustrate the existing routing protocol ORMMA-WSN and analyse various parameters viz. throughput, delay, and network load by varying the number of sensor nodes and transmission power in a multi-sink scenario. Also, comparison has been made with AODV protocol. Analysis reveals that parameters are affected with increase in number of sensor nodes, transmitter power and ORMMA-WSN outperformed in throughput and delay with respect to AODV protocol.
\end{abstract}

Keywords: WSN, ORMMA-WSN, AODV, Multi-Sink, Performance Analysis

\section{INTRODUCTION}

A wireless sensor network (WSN) consists of autonomous sensors nodes to monitor physical or environmental conditions, such as temperature, sound, pressure, etc. and to cooperatively pass their data through the network to a main location. Today wireless sensor networks have number of applications, such as industrial process and monitoring control, security control, and weather forecast so on. The WSN is built of nodes from a few to several hundred nodes. WSNs belong to the Low-Rate Wireless Personnel Area Network (LR-WPAN). Every device in the network is called sensor node. Nodes may communicate in ad-hoc way in order to extend communication range. Routing in ad-hoc networks selects the optimal path to send a message from a source to a sink. In wireless sensor network mobile nodes change frequently, thus introducing node and network mobility as new challenges. Routing algorithm must adapt to these conditions. Opportunistic routing adapts to current conditions and predicts the future behaviour. This paper aims to outline the implementation of ORMMA-WSN protocol and analysis performance on the basis of increased number of node density and transmission power. Two routing protocols will be analyzed by simulating them in OPNET simulator [1]. The rest of the paper is organized is as follows. In section 2, we give some general insights of ORMMA-WSN protocol, opportunistic routing and existing routing protocols. In section 3 background study is shown. Simulation test bed is done in section IV. In section V, we evaluate the performance results obtained from the OPNET simulations.

\section{ORMMA WSN, ROUTING PROTOC-- OLS}

Many routing protocols search a single path for routing of packet in a wireless sensor network. ORMMA-WSN is based on opportunistic routing that exploits a redundancy of nodes to transmit a packet to nodes that are available for routing. Opportunistic routing is powerful and well-suited to Wireless Sensor Networks, where nodes have intermittent connectivity and the availability of neighbour nodes for packet forwarding is disrupted. There are various Opportunistic routing protocols exist. ExOR ranks each node by number of hops. In this way it elects the forwarder node. The packet is forwarded in a sequence of nodes [2]. When the previous node finished transmitting all the data packets then ExOR selects the next forwarder. Among all the nodes in the network, the one which is closest to the destination is selected as next forwarder. This process goes on until packet reached to the destination. Next important opportunistic routing protocol is the Opportunistic Routing in Ad Hoc Networks (OPRAH) [3]. Whenever there is a dynamic network ORPAH find optimal path by using promiscuity of air interface. With the help of AODV ORPAH fill route data by not selecting single route to destination. For selecting which node act as forwarder, all nodes compete for forwarding a sent packet which are closer to the sender. It is adapted to the environment and mobility. SPIN (Sensor Protocols for Information via Negotiation), that efficiently disseminate information from node to node in the network assuming that all nodes in the network are potential basestation [4]. With the help of this the user can make query to any node and get the required information. SPIN uses the property, that the nodes that are in promiscuity use the same data and therefore there is only need to distribute the data that other nodes do not posses. A high-level name is assigned to collected data by SPIN. It performs meta-data negotiations before data is transmitted. SPIN-1 and SPIN-2 are the main versions of SPIN family protocols. Gradient-Based Routing Schurgers et al. [5] proposed another variant of directed diffusion, called Gradient-Based Routing (GBR). The main function of GBR is to remember the number of hops when the interest is diffused through the whole network. As such, each node can calculate a parameter called the height of the node, which is the minimum number of hops to reach the BS. The gradient on that link is calculated by subtracting height of node's and its neighbor node. A packet is forwarded on a link with the largest gradient. GBR used some auxiliary techniques such as data aggregation and traffic spreading in order to uniformly divide the traffic over the network. 
The Greedy Other Adaptive Face Routing (GOAFR) in [5] is a geometric ad-hoc routing algorithm combining greedy and face routing. The GOAFR works in the way that it picks the neighbor that is close to a node and act as next node for routing. However, it can be easily stuck at some local minimum, i.e. no neighbour is closer to a node than the current node. The opportunistic algorithm is implemented and simulated in the OPNET simulator. The model is built on top of a partly implemented IEEE 802.15.4 model [6].

\subsection{Overview of IEEE 802.15.4}

Open-ZB [7] is an open source implementation of IEEE 802.15.4. IEEE 802.15.4 specifies various versions from which version 1.0 of accurate simulation model of the slotted IEEE 802.15.4 is used and simulated in OPNET simulator. The IEEE 802.15.4 protocol [6] specifies the Medium Access Control (MAC) sub -layer for Low-Rate Wireless Private Area Networks (LR-WPAN). The IEEE 802.15.4 protocol targets low-data rate, low power consumption, low cost wireless networking, with typically fits the requirements of sensor networks. Both the IEEE 802.15.4 protocol and ZigBee protocol are closely related to each other. The IEEE 802.15.4 describes physical and Medium Access Control (MAC) layers. The architecture layers include Logical Link Control (LLC) that connects the MAC layer with the upper layers through. IEEE 802.5.4 also supports various network topologies such as Star, Cluster, Tree and Mesh topologies. The features of this model are real-time suitability by reservation of guaranteed time slots and collision avoidance through CSMA/CA.

\subsubsection{Physical layer}

The PHY layer contains RF Transceiver, operating with one of the specified frequency bands. The various services provided by the physical layer are data transmission service, interface to the physical layer management and access to layer management function. It also maintains a database of information. The physical layer use 3 bands with 27 channels and each band have carrier frequency of $2.4 \mathrm{GHz}, 915 \mathrm{MHz}$ and $868 \mathrm{MHz}$ s. In the latest versions of IEEE 802.15.4 two data rate patterns have been added. Activation and deactivation of radio transceiver is also another function of physical layer. Basically, there are two physical layers specified by the standards in 2003. But by the years 2009 few more were introduced which differ in their layered structure.

\subsubsection{MAC Layer}

The MAC layer provides access to the physical channel. The two services provided by MAC layer are data service and MAC management service interfacing to the MAC sublayer management entity (MLME). It enables transmission and reception of MAC protocol data units (MPDU) across the physical data service. It also contains frame validation, guarantees time slots and handles node association. This standard also defines two modes of MAC operation: beaconenabled and non-beacon enabled mode. The simulation model of IEEE 802.15.4 is being depicted by the figure number 1 .

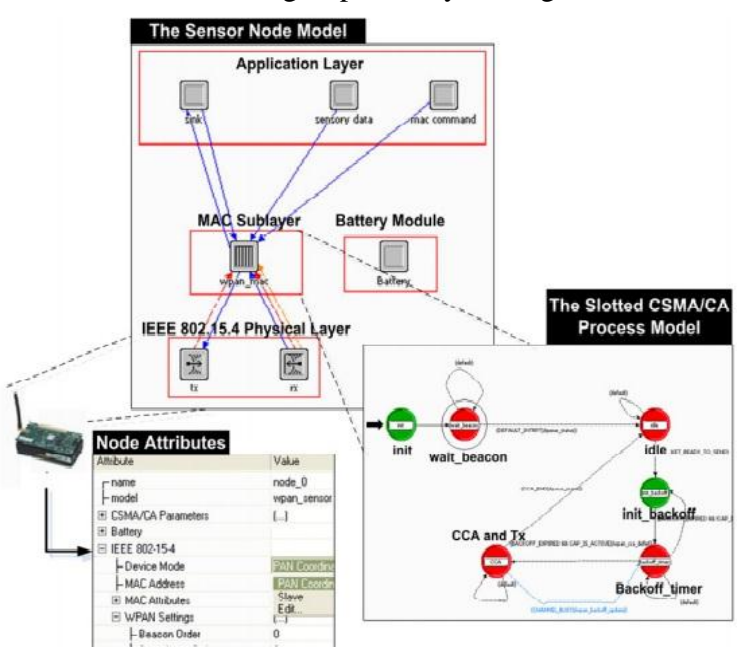

Fig. 1. Simulation model of IEEE 802.15.4 Sensor node.

\subsubsection{Beacon Enabled Mode and Slotted CSMA/CA}

In this mode the beacon frames are generated at regular interval of time. These beacon frames are generated by the PAN coordinator. Reason behind it is to synchronize various devices that are associated with the PAN coordinator and to describe superframe structure. The PAN coordinator uses the superframe structure to handle the communication between the various associated devices with it, only when it enters into beacon enabled mode. The format of the superframe is defined by the PAN coordinator and transmitted to other devices inside every beacon frame, which is broadcasted periodically by PAN coordinator. The superframe is divided into 16 equally sized slots and is followed by a predefined inactive period. As shown in figure 2, the super frame is contained in a Beacon Interval, which is bounded by two consecutive beacon frames, and includes one Contention Access Period (CAP). Beacon enabled mode supports slotted CAMA/CA mechanism. In beacon-enabled mode, access to the channel can be done only at the boundaries of this backoff period. It is a random waiting time. The slotted version of CSMA/CA algorithm starts with an initialization of scheduling variables. $\mathrm{NB}, \mathrm{BE}$ and $\mathrm{CW}$ are the three parameters of slotted CSMA/CA. The Number of Bakeoffs (NB) is the number of backoffs until the device can access the radio channel. BE is the Backoff Exponent which is sum of waiting periods before a channel can be accessed. Last is CW i.e. Contension Window a backoffs periods necessary to wait until transmission allowed.

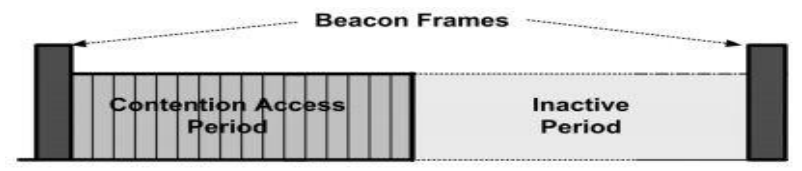

Fig. 2 The superframe structure 


\subsubsection{Non-Beacon Enabled Mode and Unslotted CSMA/CA}

Non-Beacon Enabled mode is a simplified MAC protocol. Data transmissions are done with the help of Unslotted CSMA/CA. By using Unslotted CSMA/CA any device can send data. In this the beacon frames are not send at regular interval of time but a device can request by using beacon request command frame. This mode provides various advantages such as scalability and self-organization. Also it provides simple implementation and all nodes have independent communication. Non beacon enabled mode supports ad hoc communication. There is no guarantee of delivery of data frame in this mode. Medium access control is provided by an unspotted CSMA/CA mechanism. All messages to be transmitted, with the exception of acknowledgment frames and any data frame that immediately follows the acknowledgment of a must be dispatched according to this mechanism. Non beacon enabled mode handle acknowledgement differently from beacon enabled mode. The superframe is contained in a Beacon Interval bounded by two beacon frames, and has an active period and an inactive period shown in figure 3 . The coordinator interacts with its PAN during the active period, and inters in a low power mode during the inactive period.

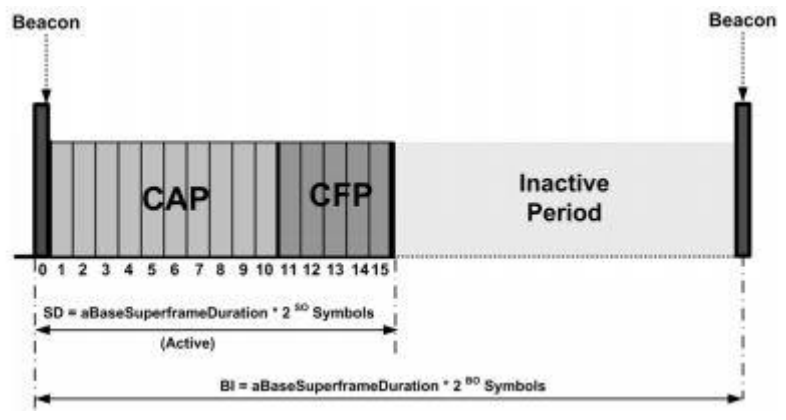

Fig. 3 Superframe Structure

\subsection{Opportunistic Routing Protocol}

The basic and fundamental idea which forms the basis of The Opportunistic Routing (OR) model is that the sensor nodes extract their mobility information from the high power beacons, which are transmitted by multiple sink nodes periodically. The nodes do not know about their global position and the node mobility is relative to the sink. When a mobile node moves away from a sink, it gives away all pending packets to the most successful mobile node, appearing in the neighbourhood. The various properties of OR protocol are calculation of Mobility Gradient (MG), obtaining node mobility information, forwarding node mobility information to neighbourhood. The RSSI values are also defined by the OR protocol which are used to obtain node's mobility information. The Received (RX) power indicates the average received signal power which identifies mobility gradient of a moving node. The network packet format defines this value. The RSSI value is indicated by the physical layer to the MAC layer which is used by the ORMMA-WSN for comparison of source and sinks nodes. Next is the Mobility
Gradient (MG) value that defines the value of node movement relative to sink. Mobility gradient can have three values: 1,0 and -1 . If $M G=1$ (positive value) then it indicates the node is moving towards the sink and $\mathrm{MG}=-1$ (negative value) indicates that it is travelling away from the sink. And $\mathrm{MG}=0$ means there is no node movement. Beacons are necessary to calculate the node mobility information. The MG value is included in the network layer packet header.

\section{BACKGROUND WORK}

A number of researchers are working in the field of Routing concepts in Wireless Sensors Network. A survey has been made on next generation cellular communication networks with a new routing algorithm Balanced Interference Routing Algorithm (BIRA) in [8]. The new network has high density base station distribution to increase the capacity and CDMA type of technology forms the base of whole network. The main feature of new routing algorithm is to take interference into account. Many experiments have been conducted so that the performance of BIRA can be compared with other traditional routing algorithms and they showed that BIRA obtains higher data rates. A new routing approach ODEUR (Opportunistic relative Distance-Enabled Unicast Routing) that is targeted to sensor networks with mobility induced intermittent connectivity and multiple alternative sinks as it is often seen in wildlife and habitat monitoring shown in [9]. The ODEUR is based on two measures: RSSI i.e. Received Signal Strength Indication and MG mobility gradient (MG). RSSI level of a node can calculate the mobility gradient MG as a measures of node movements relative to a sink. An energy consumption simulation framework for WSNs is introduced in [10]. Focus lied on the implementation of framework and the interfaces. The functionality of the framework was demonstrated by simulating the energy consumption of networks with different traffic load, routing protocols and topologies. A scheme which deploys opportunistic routing for multi-hop wireless ad-hoc networks is proposed in [11]. A reinforcement learning framework is used by this scheme so that the optimal performance can be achieved even in the absence of concrete knowledge about network model and channel statistics. The routing scheme jointly addresses the issues of learning and routing in an opportunistic context, where the transmission success probabilities characterize the network structure. A protocol which uses promiscuity of air interface to find a more optimal path for each packet in dynamic network (OPRAH) is shown in [12]. Findings are AODV protocol converges on a route that is stable throughout the simulation and OPRAH converges towards a route that is sub-optimal. It also finds a shorter path comparing to AODV path. Furthermore, the overhead of repairing the breaking routes in AODV is more comparing to OPRAH protocol. Comparing to ORPAH protocol AODV handles low, moderate and high mobility rates. It also determines unicast routes to destination within ad-hoc network. It uses an on demand approach which means that if a source node wants to send data to another then only route is establish otherwise not. AODV doesn't create any extra traffic. 


\section{SIMULATION STUDY}

Our objective is to evaluate the performance of ORMMAWSN protocol in terms of throughput, end-to-end delays by increasing node density and transmission power of nodes. The simulation set up is modelled in OPNET. The WSN network consists of mobile nodes. The number of sensor nodes (s) is larger than the number of sink nodes. The network analyzer is placed in the middle for the collection of global network statistics. The simulation area is limited to the square area e.g. $(100 * 100 \mathrm{~m})$. Sink nodes send periodic beacons. The nodes extract the mobility information from these beacons and start the transmission of personnel information Neighbour nodes obtain the forwarded personnel information about their neighbours and build the neighbourhood knowledge base. Then the data packets can be routed according to the acquired information. The simulation is done in various steps with increase number of nodes and transmission power in each step. Firstly, the number of nodes taken are $n=6$ with transmission power $\mathrm{tr}=0.001 \mathrm{w}, 0.100 \mathrm{w}$ and $2.0 \mathrm{w}$. Then the number of nodes are increased to 12 and transmission power remain the same, then the number of nodes are increased to $n$ $=24$ and lastly $\mathrm{n}=36$. The parameters that are calculated are network throughput, delay, network load, retransmission attempts. Also, the performance of ORMMA-WSN is compared with AODV protocol with node density $n=36$ and transmission power $\operatorname{tr}=2.0 \mathrm{w}$. The mobility model that is deployed is random direction, speed $=1-5 \mathrm{~m} / \mathrm{s}$, pause at the borders 5-10 s. Number of retransmissions $\mathrm{N}=2$. Packet TTL $=35$ hops.

\section{SIMULATION RESULTS}

This section discusses simulation results performed on ORMMA-WSN and AODV protocols.

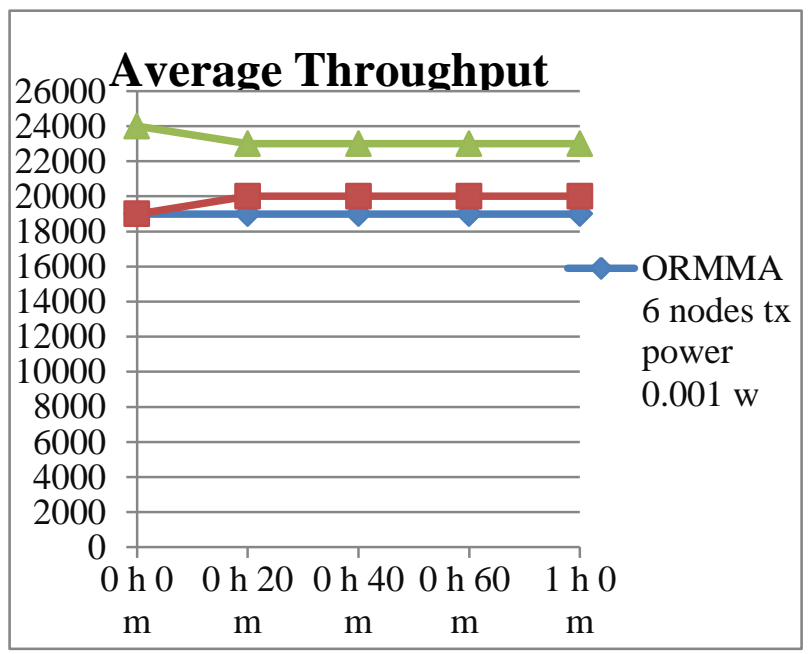

Fig. 4: Throughput of ORMMA-WSN multi-sink

From figure 4, the throughput of ORMMA-WSN is shown with node density $\mathrm{n}=6$ and three different transmission powers. As, the transmission power is increasing the throughput of ORMMA-WSN is also increasing. E.g. when tr $=0.001 \mathrm{~W}$ throughput is $19,000 \mathrm{bits} / \mathrm{sec}, \operatorname{tr}=0.100 \mathrm{~W}$ throughput is $20,000 \mathrm{bits} / \mathrm{sec}$ and when $\operatorname{tr}=2.0 \mathrm{w}$ throughput rose to $23,000 \mathrm{bits} / \mathrm{sec}$.

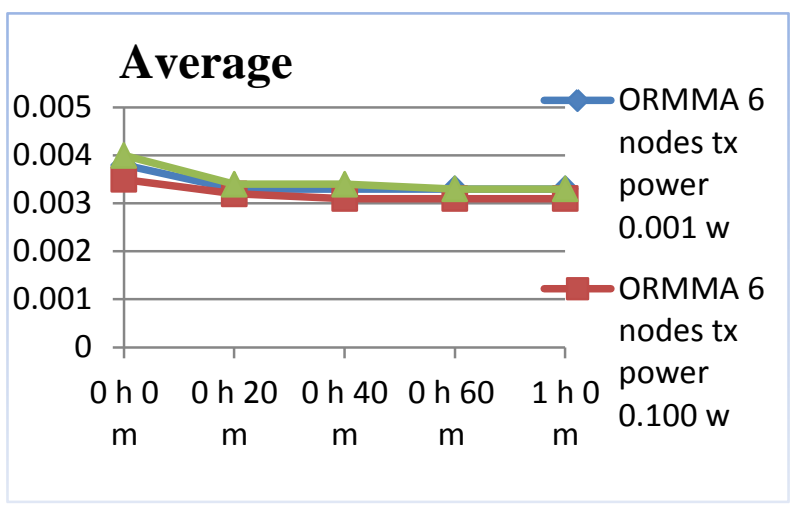

Figure 5: End-to-End Delay ORMMA-WSN

Figure 5 graph depicts, as the transmission power is increased from $\operatorname{tr}=0.001 \mathrm{w}$ to $0.100 \mathrm{w}$ there is a little decline in delay shown in red curve whereas, delay is almost same when $\operatorname{tr}=$ $0.001 \mathrm{w}=2.0 \mathrm{w}$.

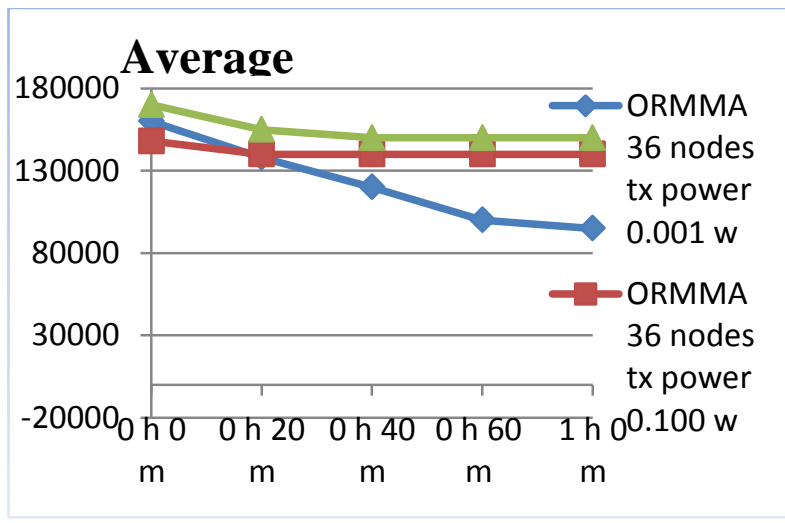

Fig. 6: Throughput ORMMA-WSN

Now the node density is increased to 36 sensor nodes. Figure 6 , showing there is a sudden decline in throughput when $\operatorname{tr}=$ $0.001 \mathrm{w}$ and throughput increased with increase in $\operatorname{tr}=2.0 \mathrm{w}$ with $150,000 \mathrm{bits} / \mathrm{sec}$. Comparing figure 1 and figure 3, it can be see that with increase in node density and transmission power there is a drastic change in throughput. It increases constantly with node density.

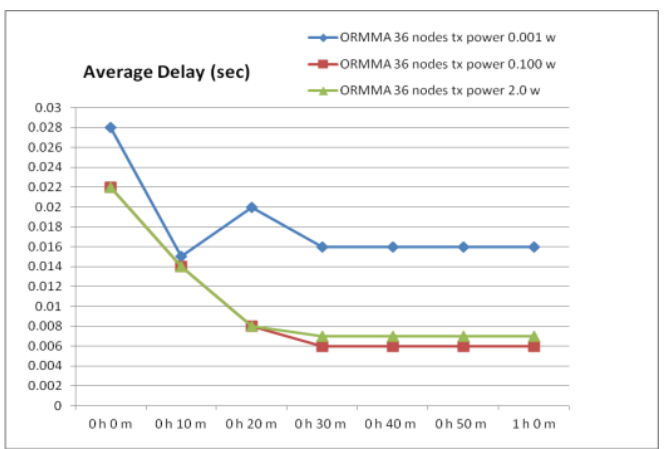

Fig 7: End-to-End delay ORMMA-WSN 
It is clearly visible from figure 7 , that by increasing the transmission power from $0.001 \mathrm{w}$ to $2.0 \mathrm{w}$ there is a gradual decline in delay e.g. when $\operatorname{tr}=0.001 \mathrm{w}$ delay is $0.016 \mathrm{sec}$. and when $\operatorname{tr}=2.0 \mathrm{w}$ delay decreased to $0.06 \mathrm{sec}$. Also, delay is decreasing when transmission power $\operatorname{tr}=0.100 \mathrm{w}$. Next the comparison is made between ORMMA-WSN and AODV routing protocols. An AODV routing model is implemented in OPNET and is provided with the MANET mobile station. AODV is based on the IP routing protocol and employs the WLAN Mac layer. In order to compare it with the ORMMAWSN, the MAC layers of both the MANET station and wireless sensor node must be the same. For this reason, PHY and IEEE 802.15.4 MAC with unslotted CSMA/CA layers are attached at the bottom of arp module. The AODV routing protocol can only work in single sink scenarios. In order to make these two models comparable single sink scenario of ORMMA-WSN must be applied too. However, evaluation of ORMMA-WSN includes multiple sinks. Both are configured to have the same scenario parameter and environmental conditions according to simulation parameters. The comparison is performed by collecting simulation statistics about throughput, end-to-end delays, and network load and retransmission attempts. Routing of data packets in AODV routing protocol is performed when the active routing path is known.

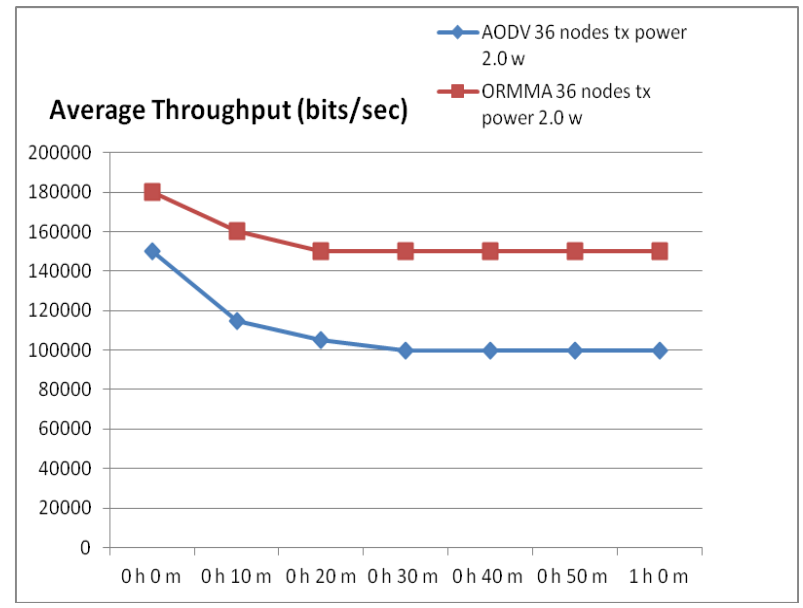

Fig. 8. Throughput of ORMMA-WSN vs AODV

ORMMA-WSN shows a large performance advantage. According to graph shown in figure 8, the throughput of ORMMA-WSN is much more comparing to AODV protocol. With node density $n=36$ nodes and TX power $=2.0 \mathrm{w}$ the throughput of ORMMA-WSN protocol is large i.e. 150,000 bits/sec. whereas throughput of AODV protocol is 100,0000 bits/sec.

Figure 9 shows the end-to-end delay in both ORMMA-WSN and AODV protocol. Comparing to AODV routing protocol, the end-to-end delay of ORMMA-WSN protocol is low.

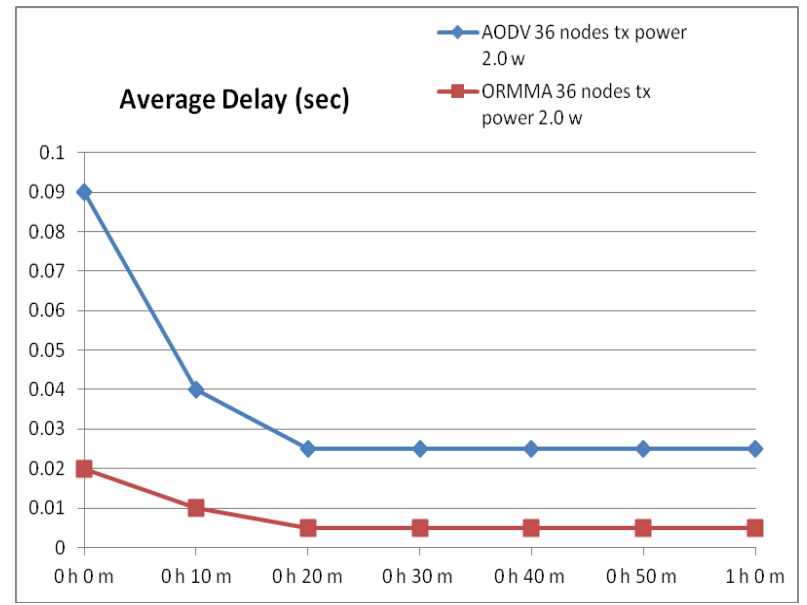

Fig. 9: Delay ORMMA-WSN vs AODV

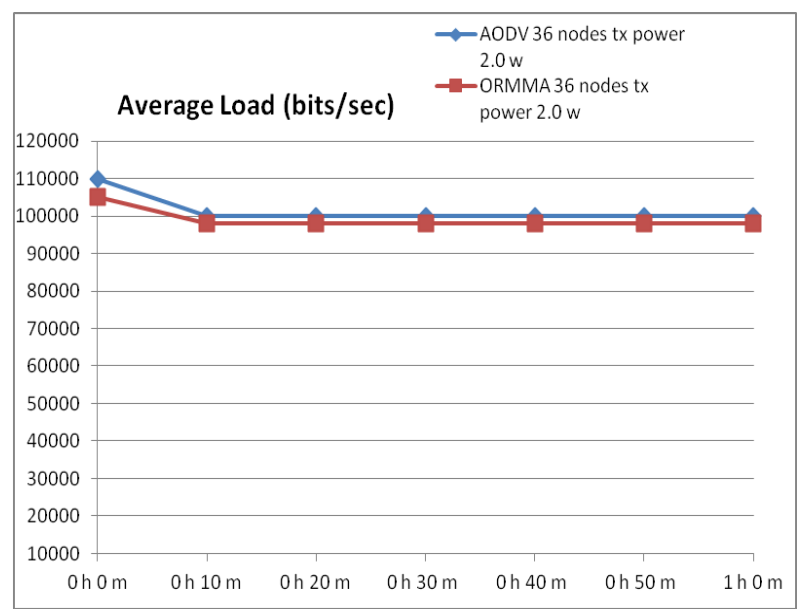

Fig. 10: Load (bits/sec) ORMMA-WSN vs. AODV

Figure 10 depicts that the network load is almost same of the two protocols ORMMA-WSN and AODV with number of nodes $\mathrm{n}=36$ and $\mathrm{tx}$ power $=2.0 \mathrm{w}$.

\section{CONCLUSION}

In this paper, we analysed the performance of ORMMA-WSN in multi sink scenario under varying number of node density and transmission power. We also compared the performance of ORMMA-WSN protocol with AODV protocol in terms of throughput, end-to-end delays and network load. Results showed that for wireless sensor network parameters like throughput, end-to-end delays, retransmission bits and load are affected greatly by different node density and transmission power. High throughput and minimum delays can be achieved in denser network with more transmission power. In second scenario ORRMA-WSN is compared with AODV in single sink scenario because AODV works only in single sink scenario. Parameters that are taken are throughput, end-to-end delays and load. It can be seen that ORMMA-WSN performs better than AODV protocol in terms of throughput and there is less end-to-end delay in ORMMA-WSN. Load is almost same for both the protocols. 
In ORMMA-WSN end-to-end ACKs and information queries from sink nodes are not covered by this work and can be considered as future work.

\section{REFERENCES}

[1] "Introduction Opnet",http://www.opnet.com.

[2] S. Biswas and R. Morris, Opportunistic routing in multihop wireless networks, ACM SIGCOMM Computer Communication Review, vol. 34, no. 1, pp. 69-74, January 2004.

[3] C. Westphal, "Opportunistic Routing in Dynamic Ad Hoc Networks: the OPRAH protocol," Mobile Adhoc and Sensor Systems (MASS), 2006 IEEE International Conference, pp. 570-573, 2006.

[4] K. Akkaya and M. Younis, "A Survey of Routing Protocols in Wireless Sensor Networks," Elsevier Ad Hoc Network Journal, vol. 3, pp. 325-349, 2004.

[5] J. Al-Karaki and A.E. Kamal, "Routing techniques in wireless sensor networks: a survey," IEEE Wireless Communications, vol. 11, pp. 6- 28, 2004.
[6] IEEE,IEEEStandard802.15.4,"http://standards.ieee.org/g etieee802/download/802.15.4-2003.pdf

[7] "Open-ZB," http://www.open-zb.net, May 2007.

[8] Fei Liu, "Routing in Multi-hop Wireless Infrastructures" university of Twent, faculty of Electrical Engineering, Mathematics and computer science(EEMCS),2009

[9] Bernd-Ludwig Wenning, Arturas Lukosious, Andreas Tmm-Giel, Carmelita Gorg1, Slobodanka Tomic, "Opportunistic Distance-aware Routing in MultiSink Mobile Wireless Sensor Networks", pp. 1-63 ,Communication Networks, University of Bremen, 28359 Bremen, Germany.

[10] Alexander Klein, Phuoc Tran-Gia, "Energy Consumption Framework for Wireless Sensor Network", University of Wuerzburg, Institute of Computer Science, Wuerzburg, Germany.

[11] A.A. Bhorkar, M. Naghshvar, T. Javidi and B.D. Rao,"An Adaptive Opportunistic Routing Scheme for Wireless Ad-hoc Networks" Department of Electrical Engineering, University of Calfornia San Diego, CA, 92093 